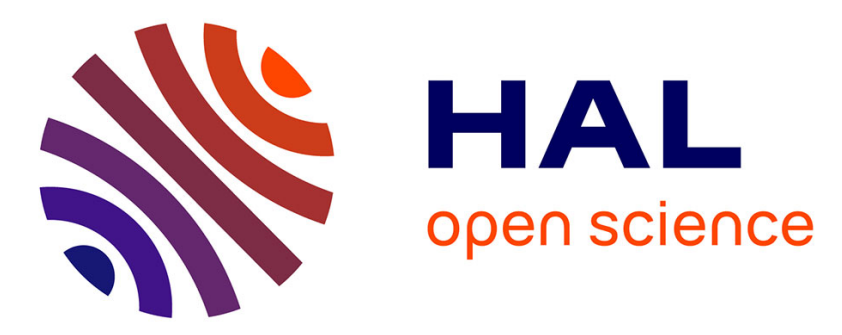

\title{
Numerical investigation of abradable coating wear through plastic constitutive law: application to aircraft engines
}

Mathias Legrand, Christophe Pierre

\section{- To cite this version:}

Mathias Legrand, Christophe Pierre. Numerical investigation of abradable coating wear through plastic constitutive law: application to aircraft engines. ASME International Design Engineering Technical Conferences \& Computers and Information in Engineering, Aug 2009, San Diego, United States. pp.907-916, 10.1115/DETC2009-87669 . hal-00413728

\section{HAL Id: hal-00413728 \\ https://hal.science/hal-00413728}

Submitted on 5 Sep 2009

HAL is a multi-disciplinary open access archive for the deposit and dissemination of scientific research documents, whether they are published or not. The documents may come from teaching and research institutions in France or abroad, or from public or private research centers.
L'archive ouverte pluridisciplinaire HAL, est destinée au dépôt et à la diffusion de documents scientifiques de niveau recherche, publiés ou non, émanant des établissements d'enseignement et de recherche français ou étrangers, des laboratoires publics ou privés.

\section{(c)(1)}

Distributed under a Creative Commons Attribution| 4.0 International License 


\title{
Numerical investigation of abradable coating wear through plastic constitutive law: application to aircraft engines
}

\author{
Mathias Legrand \\ Structural Dynamics and Vibration Laboratory, Department of Mechanical Engineering, \\ McGill University, 817 Sherbrooke St West, Montreal, Quebec H3A 2K6, Canada \\ Christophe Pierre \\ Structural Dynamics and Vibration Laboratory, Department of Mechanical Engineering, \\ McGill University, 817 Sherbrooke St West, Montreal, Quebec H3A 2K6, Canada
}

\begin{abstract}
In the field of turbomachines, better engine performances are achieved by reducing possible parasitic leakage flows through the closure of the clearance distance between blade tips and surrounding casings. Together with new technologies involving higher casing conicity for improved compression rates, direct contact is now considered as part of aircraft engines normal life. In order to avoid possibly catastrophic scenarios due to high contact efforts between the rotating and static components, implementation of abradable coatings has been widely recognized as a robust solution offering several advantages: reducing potential damage to the incurring blade as well as adjusting operating clearances, in-situ, to accept physical contact events. Nevertheless, the process of wear undergone by abradable coatings is not well understood and its consequences are still under investigation. In the present work, its macroscopic behavior is numerically approximated through a piecewise linear plastic constitutive law which allows for real time access to the current abradable layer profile. First results prove convergence in time and space of the proposed approach and show that the frequency content of the blade response is clearly affected by the presence of abradable coatings. It seems that opening the clearance between the blade tip and the casing during wear leads to large amplitudes of motion far from the usual linear conditions provided by the well-known Campbell diagrams.
\end{abstract}

Keywords: Turbomachinery; Abradable coatings; Wear; Contact dynamics; Explicit timestepping technique

\section{Introduction}

Facing a constant need of improved performances for lower operating costs, jet engine manufacturers respond with new technologies such as, amongst others, higher operating temperatures, more efficient aerodynamic designs and lightweight materials. One of the last means being addressed lies in the reduction of possible parasitic leakage flows by closing the gap between blade tips and surrounding casings. This is made possible through the implementation of abradable coatings [25] in the compressor and turbine sections (see Fig. 1 for the compressor of interest in the present study) where a minimal clearance is required. The mechanical properties of the abradable material are of primary importance as it must preserve the incurring blade-tips from damage by being reasonably soft, but also be sufficiently hard to stand very high temperatures and high-speed gas flows with inherent solid particles [12]. The role of abradable coatings is to allow for closer clearances as well as to accept physical contact events. The latter may occur through a variety of mechanisms: coincidence of vibration modes, thermal gradient in the casing, rotor imbalance due to design uncertainties, etc. Unfortunately, 


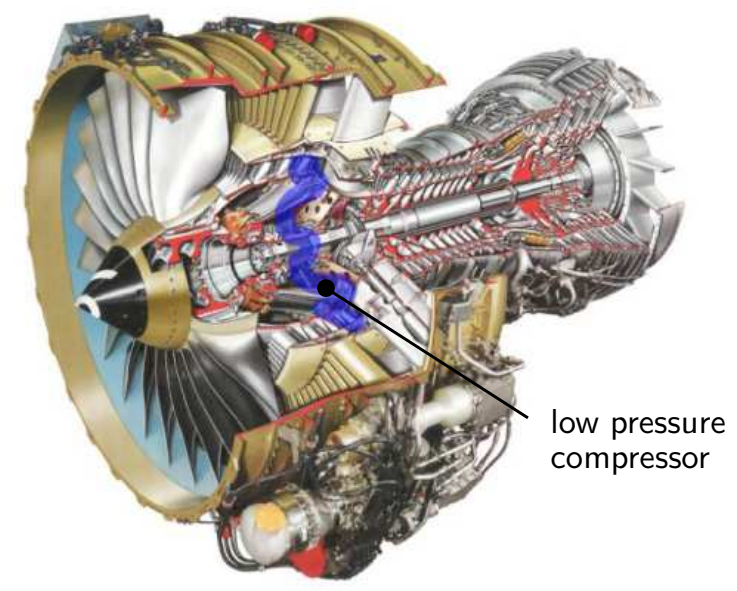

Figure 1 - Low pressure compressor. Courtesy of CFM International

it has been detected by experimental investigations that erosive wear of abradable coatings may play a significant role in the initiation of divergent behaviors such as propagating cracks in blade roots [13]. Accordingly, it seems urgent to enrich the limited current knowledge of the circumstances under which they occur [6].

Although attention has been brought in the past to the dynamics of blades in presence of direct contact $[20,11,10]$, modeling the erosion process in a macroscopic manner seems rather new $[17,9]$ even though simple analytical derivations do exist [16]. This task is rendered difficult because of the complex and coupled physical mechanisms involved such as dislocation, thermal gradients, large displacements [1] and mass removal depending on the investigated type of material. In the framework of turbomachines, where large relative displacements between contacting components together with high abradable wear rates are observed, most of the existing theoretical statements with strongly limiting assumptions do not seem relevant and easy to implement [21], even though some of them seem promising [23, 4].

It is here assumed that plasticity with its inherent abilities to represent permanent deformation in a simple fashion stands as a natural first macroscopic approach in order to account for abradable coatings erosive wear. Even though mass removal is neglected and the exact dynamic of the abradable material is not retrieved, it is thought that the behavior of the blade will be properly predicted. Furthermore, the energy transferred from the blade to the casing during contact interaction is partially dissipated because of wear: this can be captured by a plastic constitutive law.

The proposed numerical tool embeds in an explicit time marching technique, a contact detection procedure together with the calculation of internal forces and plastic deformations arising in the abradable material, thus controlling the desired amount of wear. It allows for real time access to the current profile of the abradable, supposedly key feature for detecting such critical behaviors mentioned above.

\section{Structural model and equations of motion}

\subsection{Finite element model}

The present study deals with a single rotating blade and a surrounding casing of a low pressure compressor stage, as depicted in Fig. 2. Possible gyroscopic effects, centrifugal stiffening or imbalance loads are not accounted for. The considered sector is originally discretized in 3,764 prismatic finite elements connected through 6,734 nodes. Within the well-known finite element framework under the assumption of small displacements, vector $\mathbf{u}$ stores all the displacement degrees-of-freedom of the blade and the respective mass matrix $\mathbf{M}$, damping 
matrix $\mathbf{D}$, stiffness matrix $\mathbf{K}$ and contact forces $\mathbf{F}^{\mathbf{c}}$ are built accordingly. The resulting governing equations of motion take the form:

$$
\mathbf{M u}+\mathbf{D u}+\mathbf{K u}+\mathbf{F}^{\mathrm{c}}=\mathbf{0}
$$

complemented with the usual contact constraints detailed later in the paper. Based on thor-

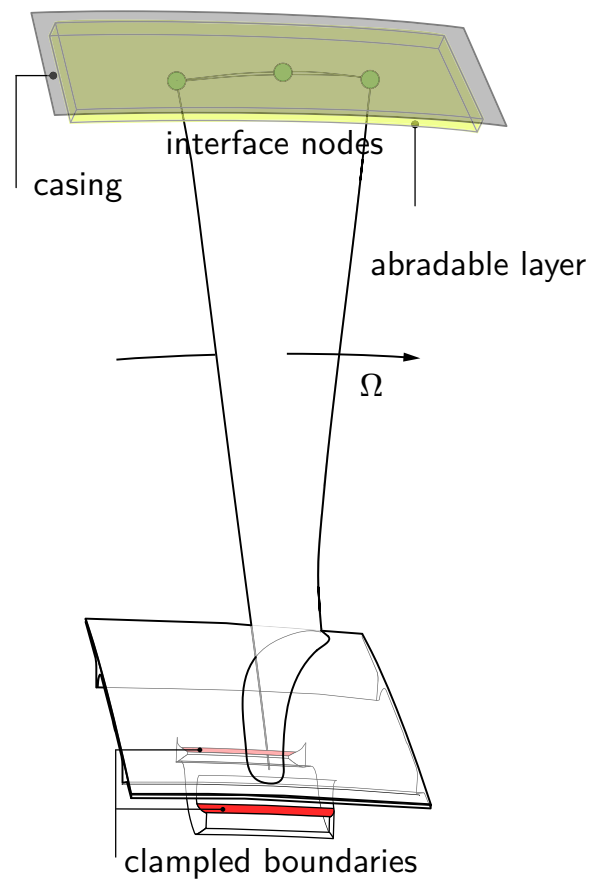

Figure 2 - Blade under investigation with the three boundary nodes undergoing contact constraints and fixed displacement boundary conditions

ough preliminary simulations, it is here admitted that the casing remains unsensitive to the contact interaction with the blade and is not modeled as a flexible component.

\subsection{Reduced-order model and component mode synthesis}

The introduced finite element model of the blade is numerically too large and leads to cumbersome computation times. It is reduced by following the Craig-Bampton procedure [3]. The original equations of motion are projected onto the new reduced-order space such as:

$$
\mathbf{u}=\left(\begin{array}{c}
\mathbf{u}_{\mathbf{f}} \\
\mathbf{u}_{\mathbf{i}}
\end{array}\right)=\left[\begin{array}{cc}
\mathbf{I} & \mathbf{0} \\
\boldsymbol{\Phi}_{\mathrm{R}} & \boldsymbol{\Phi}_{\mathrm{L}}
\end{array}\right]\left(\begin{array}{c}
\mathbf{u}_{\mathbf{f}} \\
\mathbf{q}
\end{array}\right)
$$

where the size of the reduced displacement vector $\left(\mathbf{u}_{\mathbf{f}} \mathbf{q}\right)^{\mathrm{T}}$ is substantially smaller than the size of physical displacement vector $\left(\mathbf{u}_{\mathbf{f}} \mathbf{u}_{\mathbf{i}}\right)^{\mathrm{T}}$. The dynamics of the considered blade is now seen as a full set of constraint modes whose contributions $\mathbf{u}_{\mathbf{f}}$ are the physical displacements of the interface dof, complemented with a set of component modes of contribution $\mathbf{q}$ that control the precision of the numerical simulations. As deduced from Eq. (2), the contact constraints can directly be treated in the reduced space if the anticipated contact locations are defined as interface nodes, thus avoiding permanent forward and backward mappings to the physical space. This is a major feature motivating the choice of the Craig-Bampton technique.

In this study, three nodes (leading edge, middle of the chord and tailing edge as displayed in Fig. 2) define the contact interface with the casing leading to a set of nine constraint modes: the subsequent reduced-order model should be capable of capturing complex flexural and torsional blade motions. Moreover, in order to ensure modal convergence, one hundred component modes are required: accordingly, the final size of the blade model after reduction is 
109 dof. For the sake of simplicity, Eq. (1) has undergone the projection of Eq. (2) but notations are not modified.

Since the chosen Craig-Bampton interface nodes of the blade, as such, do not contain any information about the true geometry of the blade tip, this has to be numerically included in the solution method, as illustrated in Fig. 3, in order to ensure space convergence of the abradable profile.

\section{Contact dynamics}

In order to properly define the notations later used in the proposed algorithm, derivation of the equations is conducted in the context of contact mechanics $[7,24]$ even though contact forces exerting between blade interface nodes and facing abradable coatings will not be computed explicitly.

By choice, the blade supports the master surface $\Gamma_{c}^{m}$ from which the abradable coating can be parameterized. It is then possible to find for any material point $\mathbf{x} \in \Gamma_{c}^{m}$, limited here to the interface nodes ${ }^{1}$, its closest counterpart $\overline{\mathbf{y}}$ on the abradable material slave surface $\Gamma_{c}^{s}$ :

$$
\overline{\mathbf{y}}=\arg \min _{\mathbf{y} \in \Gamma_{c}^{s}}\|\mathbf{x}-\mathbf{y}\|
$$

According to these notations, the discretized clearance between the two components can be stated:

$$
\mathbf{g}(\mathbf{x})=\mathbf{g}_{0}(\mathbf{x})+\left(\mathbf{u}^{m}(\mathbf{x})-\mathbf{u}^{s}(\overline{\mathbf{y}}(\mathbf{x}))\right) \cdot \mathbf{n}
$$

where $\mathbf{g}_{0}(\mathbf{x})$ represents the initial positive gap and $\mathbf{n}$, the outward normal to $\Gamma_{c}^{s}$. The contact conditions, referred to as the Kuhn-Tucker optimality conditions, considered in a vectorcompact form ${ }^{2}$, are such that for all $\mathbf{x} \in \Gamma_{c}^{m}$ :

$$
\mathbf{t}_{\mathrm{N}} \geqslant 0, \mathbf{g}(\mathbf{x}) \geqslant 0, \mathbf{t}_{\mathrm{N}} \mathbf{g}(\mathbf{x})=0
$$

where $\mathbf{t}_{\mathrm{N}}$ stands for the discretized contact pressure, assumed positive and acting on the contact interface. The initial clearances go from $1.5 \cdot 10^{-2} \%$ to $4.3 \cdot 10^{-2} \%$ of the blade length.

\section{Abradable constitutive law with plasticity}

As a first approach, the abradable coating is discretized with the usual one-dimensional twonode bar elements, as displayed in Fig. 3, undergoing a nonlinear plastic constitutive law. A

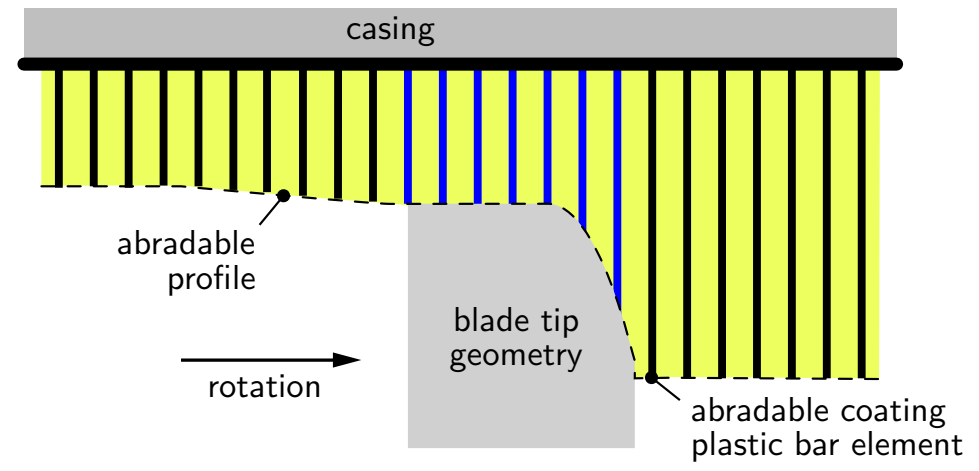

Figure 3 - Numerical profile of blade at CB interface node

\footnotetext{
${ }^{1}$ In other words, $\mathbf{x}$ is here limited to a known set of interface dof and can be seen as a set of fixed indices.

${ }^{2}$ These conditions written in a vector form have to be read coordinate by coordinate for each interface dof where contact is treated.
} 
single element is used over the thickness of the abradable layer since calculations are performed in a quasi-static compression framework only. The density, i.e. the number of bar elements along the circumference of the casing, of the abradable coating is left to investigation. By convention, strains $\varepsilon$ and stresses $\sigma$ are such that $(\varepsilon, \sigma) \in \mathbb{R}^{+} \times \mathbb{R}^{+}$. The set of admissible stresses $\mathbb{E}_{\sigma}$ is defined as follows [18]:

$$
\mathbb{E}_{\sigma}=\{(\sigma, \alpha) \in(\mathbb{R}, \mathbb{R}) \backslash f(\sigma, \alpha) \leqslant 0\}
$$

where $\alpha:[0, T] \rightarrow \mathbb{R}$ is an internal hardening variable and $f$, a yield function. It is also assumed that (1) the total strain is separated in an additive way between its elastic part $\varepsilon^{e}$ and plastic part $\varepsilon^{p}$ such as $\varepsilon=\varepsilon^{e}+\varepsilon^{p}$ and (2) the relation between elastic strains and stresses is linear $\sigma=\mathrm{E} \varepsilon^{e}$. By choice hardening is isotropic. It corresponds to a uniform (isotropic) expansion of the initial yield surface (function) with no translation. In other words, the origin of $\mathbb{E}_{\sigma}$ does not move and the hardening is linear along the plastic flow which dictates the evolution of the plastic strain $\Delta \varepsilon^{p 3}$. This leads to:

$$
f(\sigma, \alpha)=\sigma-\left(\sigma_{Y}+K \alpha\right)
$$

where $\sigma_{Y}>0$ stands for the elastic limit and $K \geqslant 0$, for the plastic modulus of the abradable material. The second assumption yields:

$$
\Delta \alpha=\Delta \varepsilon^{p}
$$

and condition on the plastic flow is implies the existence of a consistency parameter $\gamma$, such as $\Delta \varepsilon^{p}=\gamma \frac{\partial f}{\partial \sigma}$, equivalent to $\Delta \varepsilon^{p}=\gamma$ because of Eq. (7). Dual variables $\gamma$ and $f$ obey the Kuhn-Tucker conditions, complemented by the consistency condition:

$$
\gamma \geqslant 0, \quad f(\sigma, \alpha) \leqslant 0, \quad \gamma f(\sigma, \alpha)=0, \quad \gamma \Delta f(\sigma, \alpha)=0
$$

For a one-dimensional quasi-static strain formulation, more relevant to the finite element framework in displacement, the solution strategy is greatly simplified even though the nonlinear nature of the constitutive law can only be accounted for by incremental techniques. Consider an admissible state together with an imposed increment of deformation $\Delta \varepsilon$ within a purely elastic trial state:

$$
\begin{aligned}
& \sigma^{\text {trial }}=\mathrm{E} \Delta \varepsilon+\sigma \\
& \Delta \varepsilon^{p}=0 \\
& \Delta \alpha=0 \\
& f^{\text {trial }}=\sigma^{\text {trial }}-\left(\sigma_{Y}+\mathrm{K} \alpha\right)
\end{aligned}
$$

In order to ensure that the trial state belongs to $\mathbb{E}_{\sigma}, f^{\text {trial }}$ has to be tested:

- if $f^{\text {trial }} \leqslant 0$, trial and current states coincide;

- if $f^{\text {trial }}>0$, condition $(9)_{2}$ is violated and the trial state has to be corrected. The commonly adopted approach, named Return Mapping Algorithm [19], lies in the projection of the trial state on the boundary of the yield function $f=0$ together with $\gamma>0$ at constant strain. Variation of Eq. (7) yields:

$$
f=f^{\text {trial }}-\gamma(\mathrm{E}+\mathrm{K})
$$

Consequently, $f=0$ implies:

$$
\gamma=\frac{f^{\text {trial }}}{\mathrm{E}+\mathrm{K}}
$$

${ }^{3}$ Plasticity being handled in a quasi-static framework, the usual time derivative notation is replaced by a quasistatic form such as $\bullet$ becomes $\Delta \bullet$. 
and the following update is used:

$$
\begin{aligned}
& \sigma=\sigma^{\text {trial }}-\mathrm{E} \gamma \\
& \Delta \varepsilon^{p}=\gamma \\
& \Delta \alpha=\gamma
\end{aligned}
$$

The internal forces acting within each abradable element $i$ is computed following the above mentioned procedure together with integration over the elementary domain.

During a contact phase, the virtual work of the internal forces acting within the abradable coating for a virtual displacement $\boldsymbol{\delta} \mathbf{u}$ of the blade is equal by definition to the virtual work of the contact force for the same virtual displacement. By defining $\mathrm{I}=\{i \mid \mathbf{g}(i)=0\}$ (blue bar elements in Fig. 3), equilibrium of the contact forces with the internal forces can be written as:

$$
\mathbf{F}^{\mathrm{c}}=\sum_{i \in \mathrm{I}} \mathrm{A}_{i} \sigma_{i}
$$

where $\mathrm{A}_{i}$ stands for the cross-section area of an abradable element and depends on the density parameter. In other words, the role of the plastic constitutive law is twofold:

- computation of the contact forces.

- storage of the current abradable coating profile through $\varepsilon^{p}$.

\section{Time marching procedure}

\subsection{Background}

The phenomenon under investigation is inherently highly dynamically transient and making use of time stepping techniques, either implicit or explicit, seems quite natural. Anyway, solving coupled nonlinear governing Eqs. (1) and (14) is a difficult task that requires full attention. As discussed in most of the literature, explicit and implicit approaches offer specific advantages and downsides. Among the scientific community has now been reached a consensus stipulating that explicit schemes were simpler and more straightforward to implement and implicit ones were consistently better formulated. It also turns out that depending on the nonlinear terms involved in the governing equations, such as smooth cubic restoring forces versus impact or plastic evolution laws for instance, categorizing amongst the available approaches remains unclear. Nevertheless, explicit techniques do not necessitate the calculation of tangent matrices as required in Newton-like algorithms inherently used in implicit procedures. For non-smooth and non-differentiable nonlinear terms such as those mentioned above, explicit algorithms seem more relevant [22] and are adopted here. Nevertheless, great care is required in ensuring that the chosen time step does not yield erroneous results as no residual term is checked during integration.

By noting $\mathbf{u}_{n+1}$, the numerical approximation of the exact value $\mathbf{u}\left(t_{n+1}\right)$ at time $t_{n+1}=$ $t_{n}+h$ where $h$ is the time-step, the classical explicit central finite difference scheme used in this work yields:

$$
\ddot{\mathbf{u}}_{n}=\frac{\mathbf{u}_{n+1}-2 \mathbf{u}_{n}+\mathbf{u}_{n-1}}{h^{2}} \quad \text { and } \quad \dot{\mathbf{u}}_{n}=\frac{\mathbf{u}_{n+1}-\mathbf{u}_{n-1}}{2 h}
$$

\subsection{Solution method}

The contact detection as well as the internal force contribution of the abradable material are handled by employing the prediction/correction technique developed in [2] and proved to be relevant in such situations. The final algorithm is then divided into four steps: 
1. prediction, at time step $n+1$, of the displacements $\mathbf{u}$ of the blade by neglecting the presence of the abradable coating. This predicted displacement, denoted with subscript $p$, is expressed as:

$$
\mathbf{u}_{n+1, p}=\left[\frac{\mathbf{M}}{h^{2}}+\frac{\mathbf{D}}{2 h}\right]^{-1}\left(\left(\frac{2 \mathbf{M}}{h^{2}}-\mathbf{K}\right) \mathbf{u}_{n}+\left(\frac{\mathbf{D}}{2 h}-\frac{\mathbf{M}}{h^{2}}\right) \mathbf{u}_{n-1}\right)
$$

where displacements $\mathbf{u}_{n}$ and $\mathbf{u}_{n-1}$ are known.

2. determination of the gap function vector $\mathbf{g}_{n+1, p}$ between the two contacting components using Eq. (4). A search algorithm identifies all abradable elements $i \in \mathrm{I}$ being penetrated by the blade contact interface.

3. abradable internal forces computation through a deformation increment $\Delta \varepsilon$ induced by the predicted penetrations between the two bodies. Subsequent strains $\sigma_{i \in \mathrm{I}}$, hardening variables $\alpha_{i \in \mathrm{I}}$ and plastic deformations $\varepsilon_{i \in \mathrm{I}}^{p}$ are updated using the above mentioned procedure. The final vector of internal forces is calculated through Eq. (14) and the abradable profil is then updated.

4. displacements correction consistent with the calculated contact forces [10]:

$$
\mathbf{u}_{n+1}=\mathbf{u}_{n+1, p}-\left[\frac{\mathbf{M}}{h^{2}}+\frac{\mathbf{D}}{2 h}\right]^{-1} \mathbf{F}^{\mathrm{c}}
$$

The situation where the abradable coating is totally removed is not tested here since there is always abradable to be worn. It is also interesting to note that the displacement correction in Eq. (17) could be moved to Eq. (16) then including the contact force calculated at the previous time step.

\section{Results}

\subsection{Preliminaries}

Several scenarios are possible regarding the initiation of the contact between the blade and surrounding rigid casing. It is here assumed that a temperature gradient equivalent to a multi-harmonic two-nodal diameter load quasi-statically distorts the casing in order to absorb the initial clearances, as depicted in Figs. 4(a) and 4(b). The contact and wear treatment

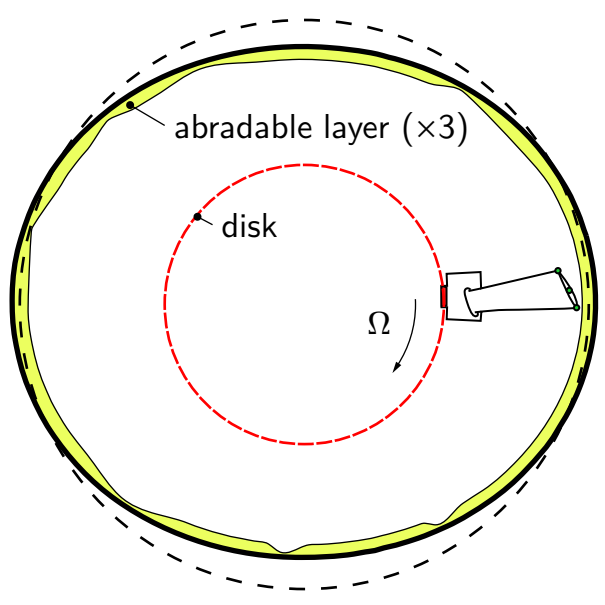

(a) Current shape of the casing

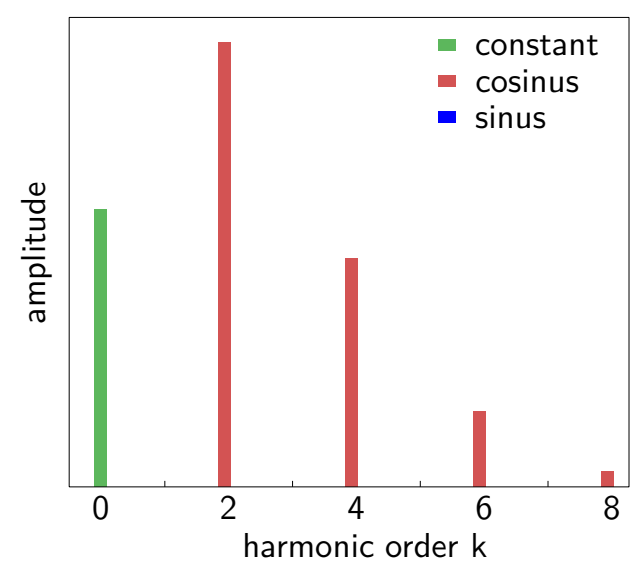

(b) Fourier transform of the shape of the casing

Figure 4 - System configuration at time $t$ 
is here limited to three boundary nodes located on the blade-tip, together with their respective abradable layer, but can be generalized to more nodes at the expense of CPU consumption.

The blade meets the casing at least twice per round depending on the calculated level of wear. Due to the rotation of the blade, the spatial harmonics of the casing shape (see Fig. 4(b)) become temporal harmonics in the rotating reference frame rigidly fixed to the blade. Accordingly, in addition to the nonlinear contact/wear participation in the blade response, the latter is expected to contain these harmonics. The wear level is governed by the plastic law parameters $\mathrm{E}, \mathrm{K}$ and $\sigma_{\mathrm{Y}}$ illustrated in Fig. 5. Specific values are not provided for the sake of brevity but two different configurations, respectively with low and high wear, are later investigated.

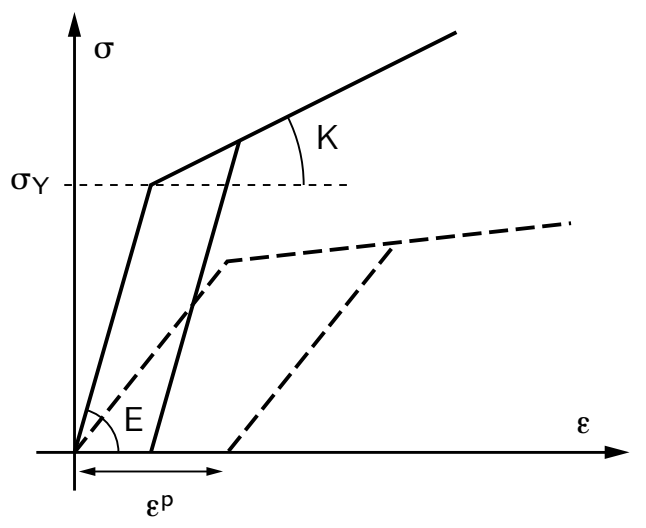

Figure 5 - Definition of the plasticity constitutive law for low ( - ) and high (- $)$ ductility of the abradable coatings

Finally, each simulation final time, denoted by $\mathrm{T}_{f}$ in the sequel, is normalized with respect to ten rounds of the blade. In a similar manner, the different depicted vibratory and wear levels and respective Fourier transforms are normalized with respect to the initial gap between interface node 1 and the casing.

\subsection{Convergence of the erosion wear law}

The convergence in space and time of the erosion wear law has to be checked before any further investigation. This is respectively achieved by increasing the density of abradable elements and by reducing the time-step of the numerical tool. Since the numerical width of the blade at the tip is constant, the profil of the abradable coating has to be independent of the mentioned density. Figure 6 depicts the final profiles of the abradable coating for different densities and same time step. Convergence is reached meaning that there exists a density threshold over which the profil becomes invariant. The sensitivity of the blade vibration to the same density parameters as in Fig. 6 is pictured in Fig. 7. The differences are almost undistinguishable and the vibratory level of the blade being a quantity of great interest in this study, we conclude that the convergence of the abradable space discretization and profil is satisfactory.

Regarding the convergence in time, Fig 8 depicts the vibratory level of interface node 1 for three different time steps. Similarly, it shows that the conditionally stable time step of the proposed numerical algorithm is reached. It is also noteworthy saying that the computation time is not highly dependent on the density as this parameter takes place in the correction step only. In the sequel, a density parameter offering the best compromise between efficiency and accuracy is chosen.

\subsection{Modal analysis and wear profile}

Illustration 9 provides the first five natural modes of vibration of the blade. Within the operating range of the low pressure compressor of interest, only the first flexural mode is expected 


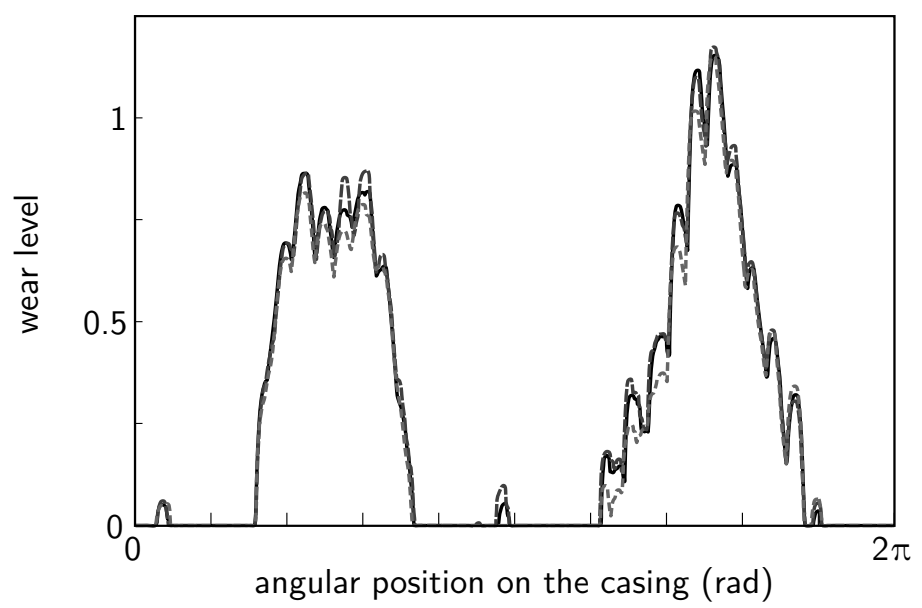

Figure 6 - Final abradable profiles for different densities and a low rotational velocity after ten rounds of the blade

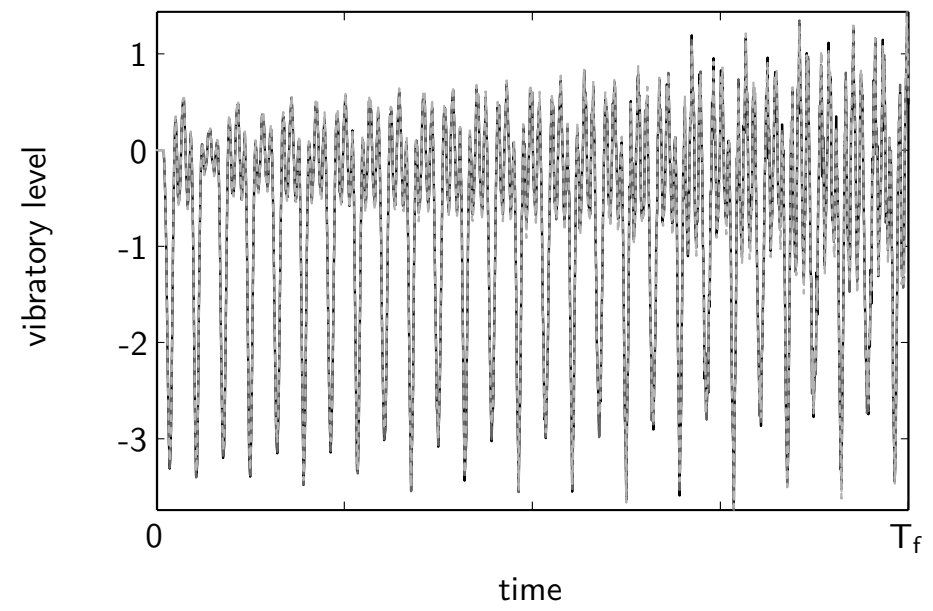

Figure 7 - Vibratory level of the blade at interface node 1 for different abradable densities

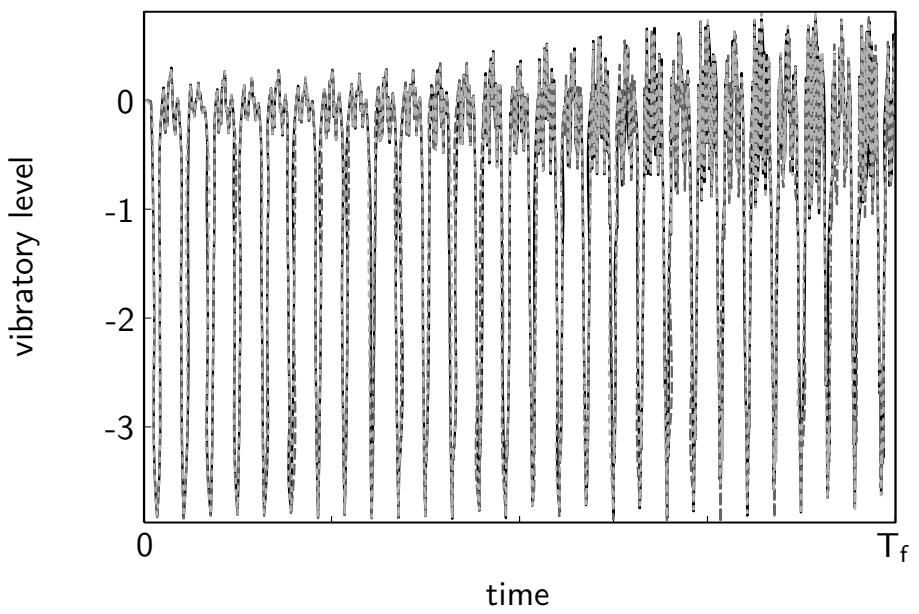

Figure 8 - Vibratory level of the blade at interface node 1 for different time step sizes

to be excited in a dangerous manner through direct contact. Its frequency is denoted by $f_{1}$ with respect to which are normalized all the frequency results and rotational velocities $\Omega$.

A series of simulations has been conducted in order to understand the sensitivity of the abradable wear law and subsequent profile to the rotational velocity $\Omega$. Beforehand, a quick modal analysis of the blade as a linear flexible structure is required to better understand the up-coming results. As the casing keeps a multi-harmonic two-nodal diameter shape during 


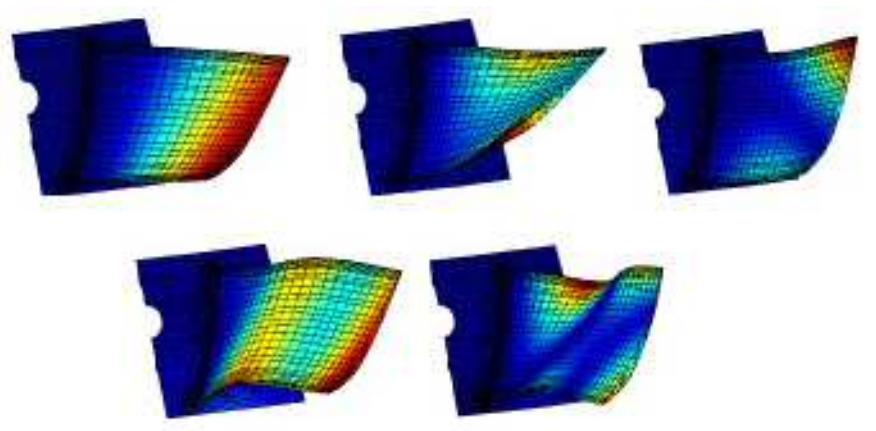

Figure 9 - First five natural modes of the blade

interaction, as highlighted in Fig. 4(b), the first frequency of the blade will be reached for $\Omega$ such as:

$$
\Omega(\mathrm{Hz})=\frac{f_{1}}{k}(\mathrm{~Hz}), \quad k=2,4,6 \ldots
$$

that describes engine-order lines crossing the first frequency of resonance of the blade. In Eq. (18), $k$ is limited to even positive integers due to the assumed shape of the casing which leads to a nonlinear harmonic external excitation due to contact with an engine-order two fundamental component ${ }^{4}$. In this context, condition (18) will thus be satisfied for lower rotational velocities than a pure linear counterpart situation with a perfect mono-harmonic twonodal diameter shape on the casing where $k=2$ would only be eligible. In what follows, we pay attention to $k=4$ and $k=6$ since $k=2$ is out of the operating range in terms of $\Omega$.

It is well known that direct unilateral contact conditions stiffen the interacting mechanical components and modify their frequency of resonance, phenomenon that can be captured by nonlinear modal analyses [5]. Accordingly, Eq. (18) could be revisited as follows:

$$
\Omega(\mathrm{Hz})=\frac{f_{1}\left(\left|\mathbf{F}^{\mathrm{c}}\right|\right)}{k}(\mathrm{~Hz}), \quad k=2,4,6 \ldots
$$

where $\left|\mathbf{F}^{\mathrm{c}}\right|$ simply refers to the amplitude of the contact forces.

Amplitude maps in Figs. 10 and 11 explicitly show the wear level in the abradable coating along the circumferential direction of the casing with respect to $\Omega$ and implicitly indicate the number of observed lobes. For low or high rotational velocities, the shape of the casing controls the wear profile since the blade does not respond in resonance to the nonlinear contact excitation and only two lobes are worn. On the contrary, when Eq. (19) is (almost) satisfied, large amplitudes of vibration are expected: this is clearly shown for $k=4$ and $k=6$ where four lobes and six lobes are distinguishable, respectively. The contact stiffening effect is well caught by the proposed algorithm since, in Figs. 10 and 11, the worn lobe highest amplitudes should be located at $\Omega=0.25(k=4)$ and $0.16(k=6)$ based on Eq. (18) but are located at $\Omega=0.31(k=4)$ and $\Omega=0.21(k=6)$ instead, see Eq. (19). In other words, the first flexural mode of the blade is excited through intermittent contact with the casing increasing the level of erosion wear for very specific $\Omega$. Nevertheless, the abradable ductility, determined by mechanical parameters $\mathrm{E}, \mathrm{K}$ and $\sigma_{\mathrm{Y}}$ do modify the conditions of interaction. As can be seen in Figs. 10 and 11 again, the rotational velocities for which the blade is in resonance are slightly different, depending on the level of wear. This will be further commented later on in the light of the analysis of the blade response frequency content. Higher harmonics for $k=8,10,12,14$ are also visible in Fig. 11 at lower rotational velocities.

The final abradable coating profile is compared in Fig. 12 for low and high wears and a rotational velocity around the respective first critical velocity numerically estimated for $k=4$. The expected four lobes are obvious.

${ }^{4}$ Other configurations with a more general shape of the casing are of great interest too in which case $k=1,3, \ldots$ 


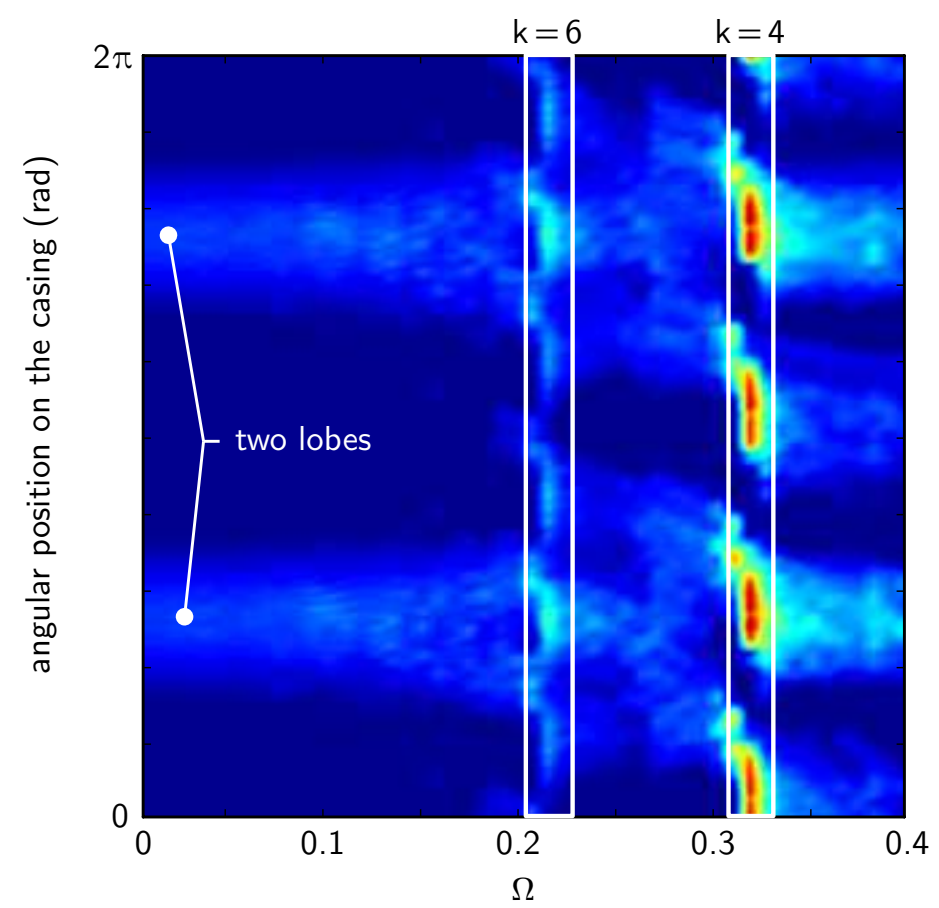

Figure 10 - Map of the final abradable profiles with respect to $\Omega$ at interface node 1 with low ductility after ten rounds of the blade

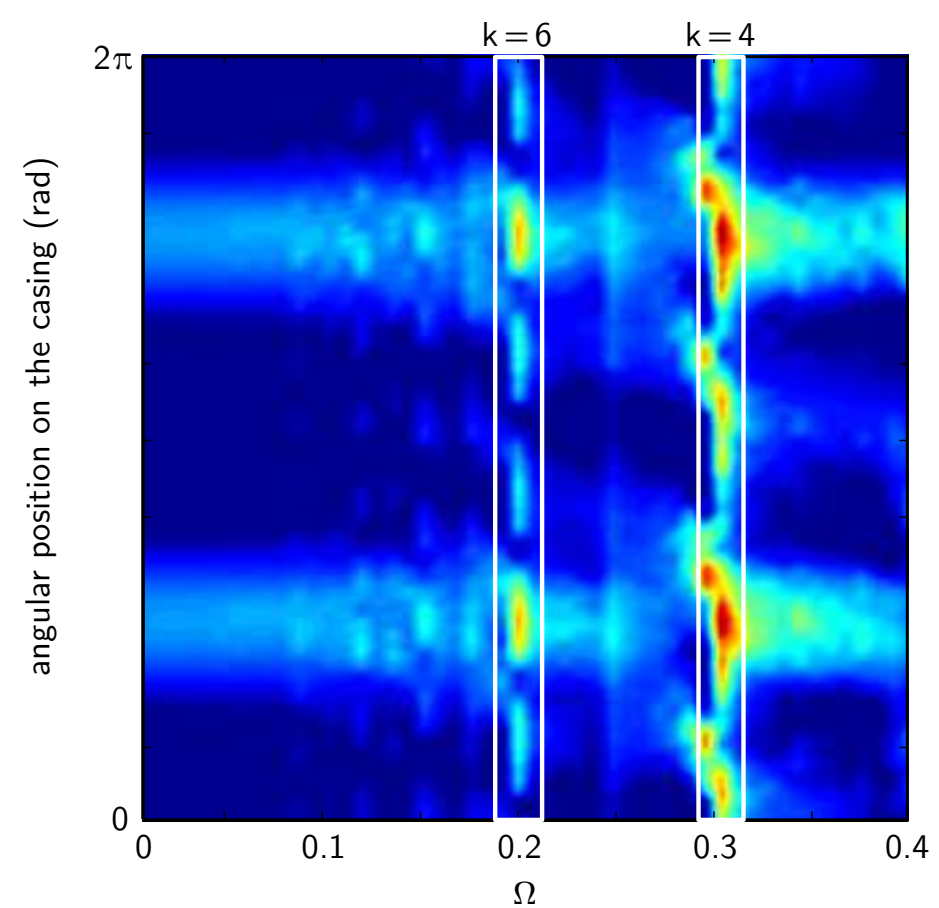

Figure 11 - Map of the final abradable profiles with respect to $\Omega$ at interface node 1 with high ductility after ten rounds of the blade

\subsection{Frequency response}

Next, a frequency content analysis of the blade response has been carried out over the operating range of the compressor. It is intended to show the effect of wear on the vibratory behavior of the blade. A Fast Fourier Transform of the blade response over ten rounds is performed for

would also arise in the solution. Situations where $k$ is not integer because of contact unilateral constraints were reported in the past but were not detected in the present study. 


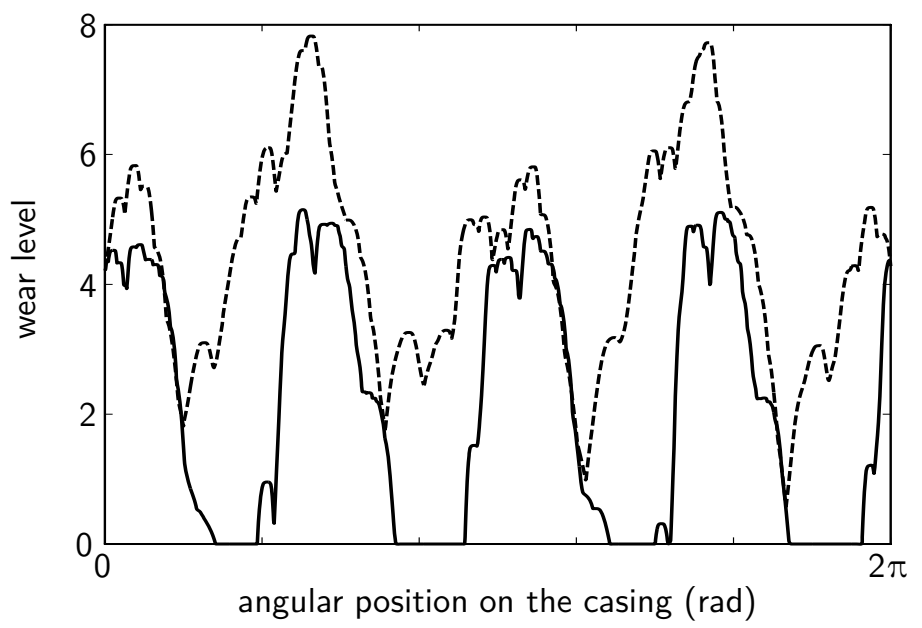

Figure 12 - Comparison of final abradable profiles for low ( - ) and high ( - - ) wear at respective critical rotational velocity for $k=4$ after ten rounds of the blade. To be complemented with Figs. 10 and 11

$\Omega=[0 ; 0.4]$. Respective results are provided in Figs. 13, 14 and 15, where $|\mathrm{FFT}(\mathbf{u})|$ denotes the Fourier spectrum of the blade response.

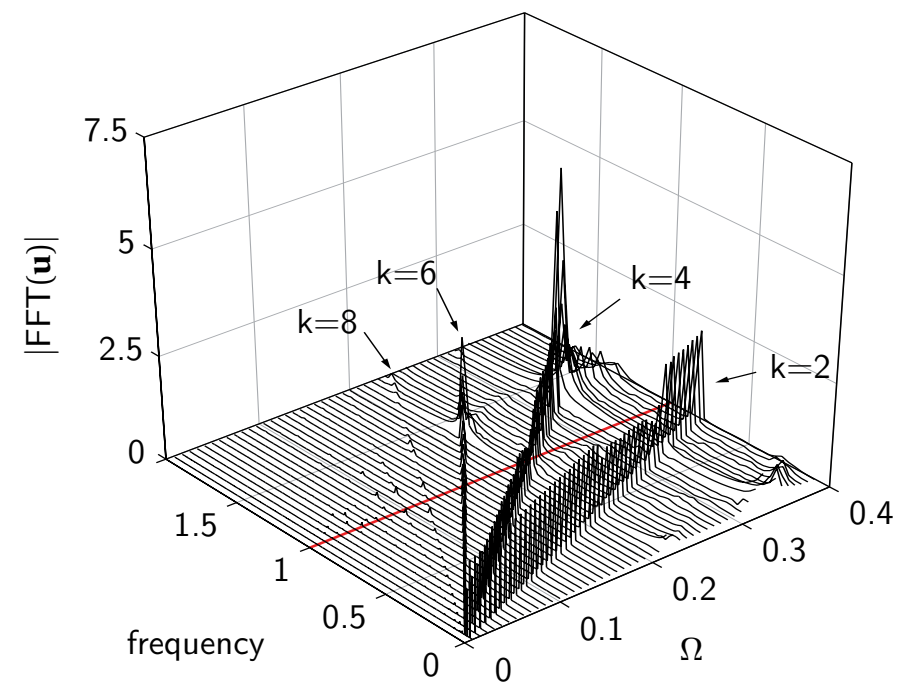

Figure 13 - Spectrum of the blade response without wear; contact forces computed through Lagrange multipliers [10]. The first natural frequency of the blade is indicated in red

Results in Figs. 14 and 15 are in perfect agreement with the final profiles depicted in Figs. 10 and 11 where the highest amplitudes are detected for $\Omega \simeq 0.21$ and $\Omega \simeq 0.31$, respectively. Most importantly, by comparing the pikes of resonance, the highest amplitudes of motion are not reached for pure unilateral contact conditions (see Fig. 13) for which the contact forces are the largest, but for a low level of wear as illustrated in Fig. 14. It means that the gap opening due to wear allows for higher displacements and are unfortunate in terms of divergent behaviors. Equivalently, pure unilateral contact conditions seem less dangerous than wear, to some extent, for the blade mechanical integrity as it inherently limits its displacements. Furthermore, very ductile abradable coatings conduct to softer contact efforts and subsequent smaller amplitudes of vibration (see Fig. 15). A compromise in the mechanical properties of the abradable material has to be found so that the useful and necessary mechanisms of wear do not lead to a structural failure of the blade.

Obviously, all the presented results strongly depend on the adopted scenario of interaction and further investigations have to be conducted to better understand the mechanisms of 


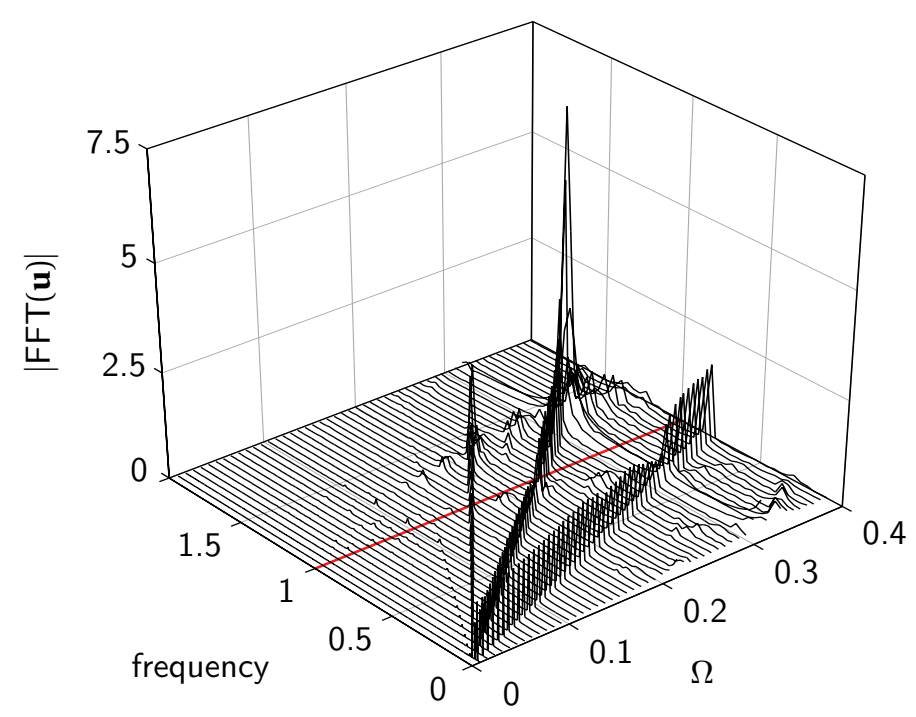

Figure 14 - Spectrum of the blade response for low ductility. The first natural frequency of the blade is indicated in red

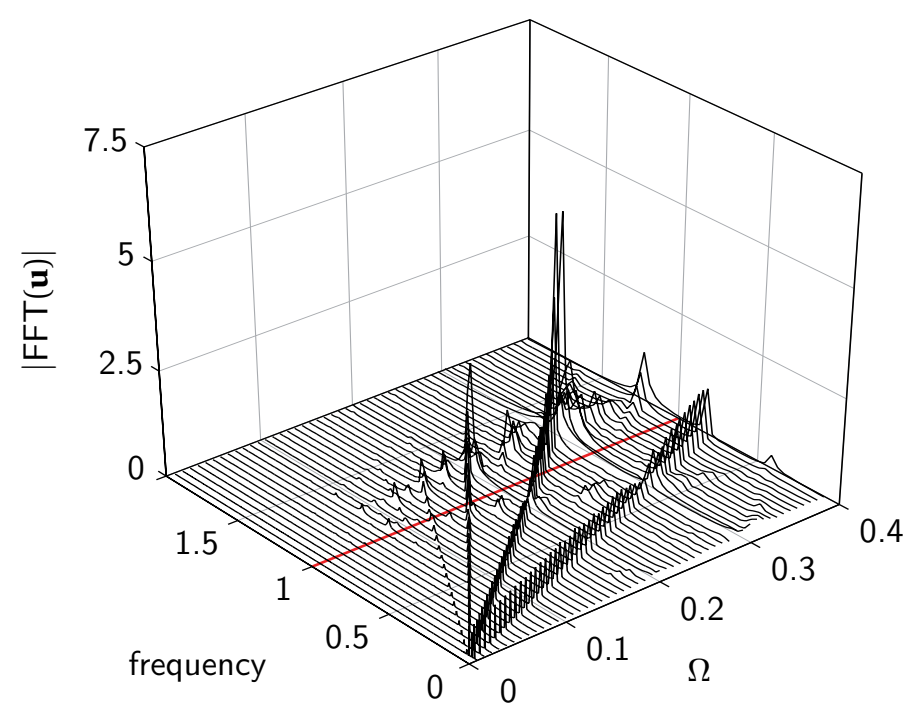

Figure 15 - Spectrum of the blade response for high ductility. The first natural frequency of the blade is indicated in red

wear and possible divergence. Nevertheless, they seem in good agreement with experimental observations about possible and unexpected severe vibration problems.

\section{Conclusion}

The emphasis of the study has been placed on the understanding of the contact interaction occurring between a blade and a surrounding casing belonging to the low pressure compressor of an aircraft engine. The study more specifically focuses on modeling in a realistic and macroscopic fashion the erosion wear law of abradable coatings which are used to soften the direct contact between interacting components. Our numerical tool couples an explicit timestepping procedure to a plastic constitutive law that approximates wear.

First results show that the developed model provides understandable and consistent physical results and is consequently fully reliable and may be used for further investigations with friction. They also exhibit unexpected complex behaviors of the blade. It seems that by opening the operating clearance between the blade tip and the casing, larger motions may be ex- 
pected far from the usual interaction conditions provided by the well-known Campbell diagrams.

In order to better estimate the wear parameter, comparisons with experimental results such as the ones presented in $[14,15]$ have to be scheduled in a near future. Other approaches in the form of frequency-domain formulated nonlinear normal modes may be relevant for faster parameter studies [8].

\section{Acknowledgement}

Thanks go to Snecma for its technical and financial support. This work takes place in the framework of the MAIA mechanical research and technology program sponsored by CNRS, ONERA and SAFRAN Group.

\section{References}

[1] Kevin T. Andrews, M. Shillor, S. Wright, and A. Klarbring. A dynamic thermoviscoelastic contact problem with friction and wear. International Journal of Engineering Science, 35 (14):1291 - 1309, 1997. ISSN 0020-7225. doi:10.1016/S0020-7225(97)87426-5.

[2] N. Carpenter, R. Taylor, and M. Katona. Lagrange constraints for transient finite element surface contact. International Journal for Numerical Methods in Engineering, 32:130 - 128, 1991. doi:10.1002/nme.1620320107.

[3] R.R. Craig and C.C. Bampton. Coupling of substructures for dynamics analyses. AIAA Journal, 6(7):1313 - 1319, 1968. doi:10.2514/3.4741.

[4] A. Dmitriev, A. Smolin, V. Popov, and S. Psakhie. A multilevel computer simulation of friction and wear by numerical methods of discrete mechanics and a phenomenological theory. Physical Mesomechanics, 12(1-2):11 - 19, 2009. ISSN 1029-9599. doi:10.1016/j.physme.2009.03.002.

[5] D. Jiang, C. Pierre, and S. Shaw. Large-amplitude non-linear normal modes of piecewise linear systems. Journal of Sound Vibration, 272:869 - 891, 2004. doi:10.1016/S0022-460X(03)00497-8.

[6] J. Jiang, J. Ahrens, H. Ulbrich, and E. Scheideler. Contact model of a rotating rubbing blade. In Proceedings of the 5th International Conference on Rotor Dynamics of the IFTOMM, pages 478 - 489, Darmstadt, Germany, June 1998.

[7] T. Laursen. Computational contact and impact mechanics - Fundamentals of modeling interfacial phenomena in nonlinear finite element analysis. Springer-Verlag, Heidelberg, 2002. ISBN 3540429069.

[8] D. Laxalde, L. Salles, L. Blanc, and F. Thouverez. Non-linear modal analysis for bladed disks with friction contact interfaces. In Proceedings of GT2008 ASME Turbo Expo 2008: Power for Land, Sea and Air, number GT2008-50860, Berlin, Germany, June 2008.

[9] M. Legrand. Modèles de prédiction de l'interaction rotor/stator dans un moteur d'avion. PhD thesis, École Centrale de Nantes, France, 2005. URL http://tel.archives-ouvertes.fr/tel-00011631/fr/.

[10] M. Legrand, C. Pierre, P. Cartraud, and J.-P. Lombard. Two-dimensional modeling of an aircraft engine structural bladed disk-casing modal interaction. Journal of Sound and Vibration, 319(1-2):366 - 391, 2009. ISSN 0022-460X. doi:10.1016/j.jsv.2008.06.019. URL http://hal . archives-ouvertes.fr/hal-00328186/fr/. 
[11] N. Lesaffre, J.-J. Sinou, and F. Thouverez. Contact analysis of a flexible bladed-rotor. European Journal of Mechanics - A/Solids, 26(3):541 - 557, 2007. ISSN 0997-7538. doi:10.1016/j.euromechsol.2006.11.002.

[12] X. Ma and A. Matthews. Investigation of abradable seal coating performance using scratch testing. Surface and Coatings Technology, 202(4-7):1214 - 1220, 2007. ISSN 0257-8972. doi:10.1016/j.surfcoat.2007.07.076.

[13] C. Padova, J. Barton, M. Dunn, and S. Manwaring. Experimental results from controlled blade tip/shroud rubs at engine speed. Journal of Turbomachinery, 129(4):713-723, 2007. doi:10.1115/1.2720869.

[14] C. Padova, M. Dunn, and J. Barton. Casing treatment and blade tip configuration effects on controlled gas turbine blade tip/shroud rubs at engine conditions. In Proceedings of GT2008 ASME Turbo Expo 2008: Power for Land, Sea and Air, number GT2008-50112, Berlin, Germany, June 2008.

[15] F. Peyraut, J.-L. Seichepine, C. Coddet, and M. Hertter. Finite element modeling of abradable materials - identification of plastic parameters and issues on minimum hardness against coating's thickness. Internationl Journal for Simulation and Multidisciplinary Design and Optimization, 2:209 - 215, 2008. doi:10.1051/ijsmdo:2008028.

[16] E. Rabinowicz. Friction and wear of materials. John Wiley \& Sons, Inc., New-York, 1965. ISBN 978-0-471-83084-9.

[17] L. Salles, L. Blanc, F. Thouverez, and A. Gouskov. Analysis of a bladed disk with friction and fretting-wear in blade attachments. In Proceedings of GT2008 ASME Turbo Expo 2008: Power for Land, Sea and Air, number GT2008-51112, Berlin, Germany, June 2008.

[18] J. Simo and T. Hughes. Computational inelasticity. Springer, 1998. ISBN 0387975209.

[19] J.C. Simo and R.L. Taylor. A return mapping algorithm for plane stress elastoplasticity. International Journal for Numerical Methods in Engineering, 22:649 - 670, 1986. ISSN 1097-0207. doi:10.1002/nme.1620220310.

[20] Sunil K. Sinha. Non-linear dynamic response of a rotating radial timoshenko beam with periodic pulse loading at the free-end. International Journal of Non-Linear Mechanics, 40(1):113 - 149, 2005. ISSN 0020-7462. doi:10.1016/j.ijnonlinmec.2004.05.019.

[21] N. Strömberg, L. Johansson, and A. Klarbring. Derivation and analysis of a generalized standard model for contact, friction and wear. International Journal of Solids and Structures, 33(13):1817 - 1836, 1996. ISSN 0020-7683. doi:10.1016/0020-7683(95)00140-9.

[22] D. Vola, E. Pratt, M. Raous, and M. Jean. Consistent time discretization for a dynamical contact problem and complementarity techniques. Revue Européenne des Éléments Finis, 7:149 - 162, 1998.

[23] Y.-F. Wang and Z.-G. Yang. Finite element model of erosive wear on ductile and brittle materials. Wear, 265(5-6):871 - 878, 2008. ISSN 0043-1648. doi:10.1016/j.wear.2008.01.014.

[24] P. Wriggers. Computational contact mechanics. Wiley, 2002. ISBN 3540326081.

[25] M. Yi, J. He, B. Huang, and H. Zhou. Friction and wear behaviour and abradability of abradable seal coating. Wear, 231(1):47 - 53, 1999. ISSN 0043-1648. doi:10.1016/S0043-1648(99)00093-9. 